\title{
FACTORES QUE INCIDEN EN EL NIVEL DE POBREZA \\ DE LAS FAMILIAS CAMPESINAS EN LAS COMUNIDADES DE LA \\ MONTAÑITA UNO Y LA MONTAÑITA DOS, MUNICIPIO \\ DE TISMA, MASAYA
}

Yvette Sánc hez Medioroz ${ }^{1}$, Elgin Antonio Vivas Viachica ${ }^{2}$

${ }^{1}$ Direccion de docencia, Universidad Nacional Agraria, Ivete.sanchez@una.edu.ni,

Decano de la Facultad de Desarrollo Rural, Universidad Nacional Agraria, elgin.vivas@una.edu.ni

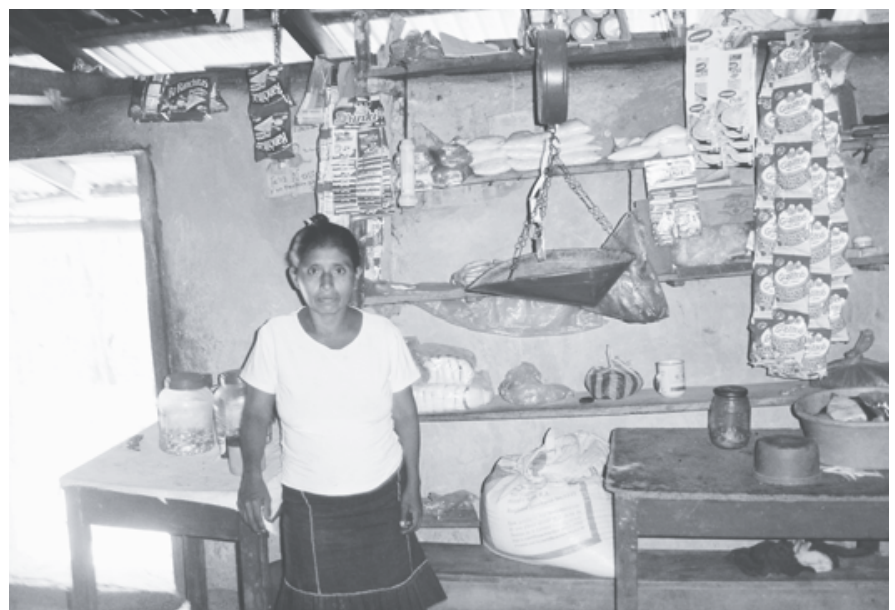

\section{RESUMEN}

Este estudio se realizó en las comunidades de La Montañita 1 y La Montañita 2 del Municipio de Tisma, Departamento de Masaya, en el año 2002. Se aplicaron 30 encuestas por comunidad. Las comunidades de "La Montañita 1" y "La Montañita 2" del Municipio de Tisma, Departamento de Masaya tienen una población mayoritariamente analfabeta, un factor que incide negativamente en elevar el nivel de vida de las familias campesinas y se constituye en una dificultad para emprender acciones de desarrollo y erradicación de la pobreza a corto plazo. Un segmento las poblaciones de estas comunidades tienen nivel académico de primaria incompleta, siendo potencialmente analfabetos; y otro porcentaje (13.2\% y $27.1 \%$ Montañita 1 y 2 respectivamente) nunca han recibido ningún tipo de formación. La capacitación de estas comunidades ha sido insignificante, debido a que el $89.1 \%$ no ha recibido ningún tipo de capacitación y solamente el $10.9 \%$ dice haber recibido. De las personas que han recibido capacitación, el 4\% lo ha hecho solamente una vez, esto nos indica que en estas comunidades los proyectos no han priorizado la capacitación como estrategia de desarrollo y mejoramiento del nivel de vida. Es importante mencionar que el $47.7 \%$ dice que nadie ha llegado a capacitar y el $16.7 \%$ piensa que no tiene tiempo disponible para participar en este tipo de actividades. Otro factor importante a considerar en el mejoramiento del nivel de vida de las comunidades rurales es la organización comunitaria,
ABSTRACT

This study was carried out at the communities La Montañita 1 and La Montañita 2 in Tisma, department of Masaya, in year 2002. Thirty surveys were carried out in each community. The communities have a high illiterate population. In addition, the illiteracy is a factor that negatively affects the standard of life of the family farmers and is a barrier that avoids the eradication of the poverty in a short term. A segment of the populations of these communities have an incomplete primary academic level, being potentially illiterates; and another percentage (13, $2 \%$ and 27,1 \% at La Montañita 1 y 2 respectively) has never received any kind of education. The education of these communities has been insignificant due to the fact that $89.1 \%$ has not received any type of education and only the $10.9 \%$ express to have received it. Four percent of the inhabitants have received education just once. This indicates that in these communities development projects have not prioritized training and education. Education has been not considerate as a development strategy and improvement of the living standard. Forty seven percent of the interviewed says that none organization, institution, and trainers, has come to offer training and 16, 7 think that they do not have the time to receive such training. Another factor to consider in the improvement of the living standard of the rural communities is the communitarian organization. In the study, it was noted that $85.7 \%$ of the population did 
en las poblaciones estudiadas se logro observar que el $85.7 \%$ no pertenecía a ningún tipo de organización y el 14.3\% no tuvo vínculos con alguna organización. Además, el 85.5\% dice no haber ningún tipo de beneficio.

Palabras clave: Pobreza, Analfabetismo y Nivel de Vida not belong to any type of organization, and that $14.3 \%$ did not have any kind of relationship with any organization. In addition, 85,5\% express not to receive any kind of benefit.

Keywords: Poverty, Illiteracy, Standard of living
$\mathrm{U}$ no de los problemas globales de la humanidad concierne a la pobreza e inequidad de de un total de 6,000 millones de habitantes, 2,800 millones, casi la mitad, viven con menos de US\$2 diarios, y 1,200 millones, una quinta parte, con menos de US\$1 al día. En los países ricos, los niños que no llegan a cumplir cinco años son menos de uno de cada 100, mientras que en los países más pobres una quinta parte de los niños no alcanza esa edad. Asimismo, mientras que en los países ricos menos del 5\% de todos los niños menores de cinco años sufre de malnutrición, en las naciones pobres la proporción es hasta del 50\%.

El ingreso promedio en los 20 países más ricos es 37 veces mayor que el de las 20 naciones más pobres; esta brecha se ha duplicado en los últimos 40 años.

Esta realidad global, obliga a los países con economías dependientes a buscar causas y factores que influyen en los niveles de pobreza de las comunidades rurales, tema que está siendo abordado por las diferentes cumbres mundiales de líderes políticos y organizaciones mundiales promotoras del desarrollo. En este contexto, el gobierno de Nicaragua tiene un plan de erradicación de la pobreza, el que no tendrá mayor impacto si no se conocen las principales causas por regiones geográficas y comunidades, que permitan priorizar las acciones que serán impulsadas por los distintos programas de mejoramiento del nivel de vida de las comunidades rurales.

Existen diferentes definiciones de pobreza, en este caso se retoma el concepto de Renzi (1996) que la define como “... el reflejo de la inequidad en la distribución del ingreso y de las relaciones de poder entre las personas y de manera particular entre los sexos. La pobreza es la manifestación de carencia materiales y otras que no son tangibles. La tendencia y dinámica de la pobreza tiene componentes sociales, culturales, psicológicos y políticos. Algunas de las características de la pobreza, se heredan a la descendencia mas allá de la inmediata, como las deficiencias nutricionales”.

\section{METODOLOGÍA}

Este estudio se realizó en las comunidades de La Montañita 1 y La Montañita 2 del Municipio de Tisma, Departamento de Masaya, en el año 2002. Se aplicaron 30 encuestas por comunidad, para un total de 60 encuestas llenadas.
El estudio tiene como objetivo: Identificar las principales causas del nivel de pobreza en dos comunidades rurales del Departamento de Masaya, Municipio de Tisma: La Montañita 1 y La Montañita 2, para el año 2002.

El estudio se realizó en las comunidades de La Montañita 1 y La Montañita 2 del Municipio de Tisma, Departamento de Masaya. Se procedió con los siguientes pasos metodológicos: Estudio de distintas metodologías utilizadas por organismos nacionales e internacionales para la medición de la pobreza: Programa de las Naciones Unidas para el Desarrollo, INEC (Actual INIDE) y FIDEG; rediseño de un instrumento para medición de pobreza en comunidades rurales; validación del instrumento; capacitación de encuestadores; aplicación de la encuesta en una muestra de 60 hogares (30 por comunidad); procesamiento y análisis estadístico en el programa SPSS, el análisis estadístico consiste en estadística descriptiva de frecuencias y agrupaciones.

\section{RESULTADOS Y DISCUSIÓN}

El origen las comunidades de la Montañita 1 y La Montañita 2, del Municipio de Tisma Departamento de Masaya, desde el punto de vista urbano-rural; se encontró que el 96.2\% de la población de La Montañita 1 es de origen rural y solamente el 3.8\% tiene origen urbano, comportamiento de la población en La Montañita 2 es bastante similar dado que el 83.0\% población proviene del sector rural y $17,0 \%$ del urbano; contrario a la tendencia nacional del $58.7 \%$ urbana y $43.3 \%$ rural comparable con la tendencia mundial del proceso de urbanización de la población.

En las poblaciones estudiadas se encontró que tienen un comportamiento estable desde el punto de vista de la movilidad geográfica, la migración no representa un problema serio (aún) ya que el $97.4 \%$ de sus pobladores ha vivido de forma permanente en la comunidad y solamente el $2.6 \%$ de sus habitantes han emigrado a otro lugar o ha llegado de otro lugar. Este comportamiento se valora de forma positiva para impulsar proyectos de desarrollo, ya que se estaría trabajando en presencia de una población estable desde el punto de vista migratorio. 
Análisis por edad. Las personas de edades comprendidas entre los 15 y los 25 años se definen como jóvenes y constituyen más de un quinto de la población mundial y en una importante fuerza considerar en los procesos de desarrollo social. El grupo etario de 15 a los 25 años es decisivo para el desarrollo familiar y de la sociedad, dado que es el período que se pasa de ser miembro de una familia a formar una propia, de la preparación a la aplicación de conocimientos.

Es importante mencionar que según datos de la FAO, el 85\% de los jóvenes viven en países en desarrollo y más de la mitad viven en zonas rurales. "Ante la falta de oportunidades de educación y empleo en el medio rural, muchos jóvenes han emigrado a las ciudades .Como resultado de ello, las zonas rurales quedan privadas de un futuro, mientras que las zonas urbanas se ven invadidas por jóvenes que no están preparados para las dificultades y las exigencias del empleo urbano”.

En el caso particular de la Comunidad de La Montañitas 1 y La Montañita 2, se encuentra una población mayoritariamente joven, el porcentaje acumulado hasta los 25 años es de $66.5 \%$ y 67.4 respectivamente, lo cual es un comportamiento que tiene sus ventajas y desventajas, debido a que representa un potencial para el crecimiento de la población y para el crecimiento de la demanda de servicios básicos. años que es de $66.5 \%$, lo que se valora como una ventaja inmediata para realizar algunas intervenciones de desarrollo con este estrato poblacional, se debe prestar especial atención a está condición, de tal forma que no se constituya en una fuente de crecimiento geométrico de la población pobre sin ninguna perspectiva. Esto se corrobora en la cifra del 58.3\% que ubican en la categoría de hijos, lo cual profundiza los niveles de dificultades socioeconómicas de los hogares de estas comunidades.

Análisis por sexo. La estructura de la población según el sexo, en las comunidades de La montañita 1 y La Montañita 2, difiere un poco a las cifras nacionales registradas; ya que en estas comunidad la población masculina es ligeramente mayor, registrándose el $50.4 \%$ en La montañita 1 y 50.2\% en La Montañita 2; a nivel nacional, según el censo 2005, el $48.6 \%$ de la población son hombres. Sin embargo, la diferencia no se considera como significativa para el desarrollo de proyectos que tengan como objetivo disminuir los niveles de pobreza e inclinarlos con una visión de genero dirigida especialmente hacia hombres, más bien se considera importante desarrollar programas dirigidos hacia mujeres que impacten de forma directa sobre el nivel de vida familiar.

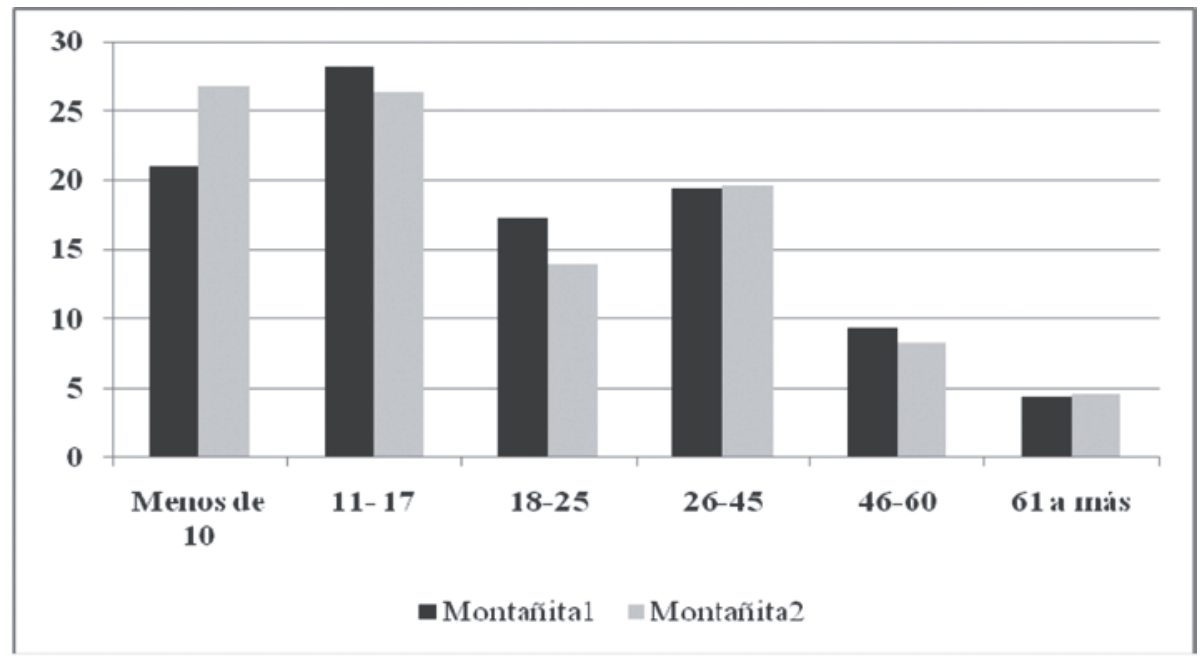

Figura 1. Distribución porcentual de la población de la montañita 1 y 2 por edad etaria.

La tendencia por grupos de edades, que se evidencian en la figura 1, muestra que la concentración de población joven en las comunidades estudiadas es mayor que a nivel nacional, de acuerdo con el censo 2005, del $49.00 \%$ de la población del país es menor de 25 años.

El 64.5\% de la población registró el estado civil soltero (a) correspondiéndole a la población menor de 25
Análisis por nivel educativo. El nivel educativo, se considera factor clave en el nivel de vida familia y como forma de combatir los niveles de pobreza de las comunidades; existe un consenso mundial que el nivel educativo es un factor determinante para el desarrollo de los pueblos en la batalla contra la pobreza, partiendo que existen tres tipos de pobreza: pobreza económica, 
pobreza intelectual y pobreza espiritual. Donde la pobreza intelectual incide de forma directa sobre la pobreza económica y espiritual; en este sentido Alvin Toffler (1990) afirma que "el conocimiento en sí mismo resulta ser no sólo la fuente del poder de más calidad, sino también el ingrediente más importante de la fuerza y la riqueza. En otras palabras, el conocimiento ha pasado de ser un accesorio del poder del dinero y del poder del músculo, a ser su propia esencia”. agregarle que un $22 \%$ no sabe leer, un $17.2 \%$ no sabe contar y un 23.8\% no sabe escribir.

Cuando se puntualiza en las razones del por qué no estudian, se encontró como respuesta más frecuente la de "no se matriculó" y un buen porcentaje de 18.0\% dijo "por trabajo". Después de haber visualizado un panorama general acerca de los niveles educativo en estas comunidades, observamos que el proceso de capacitación de estas comunidades es insignificante,

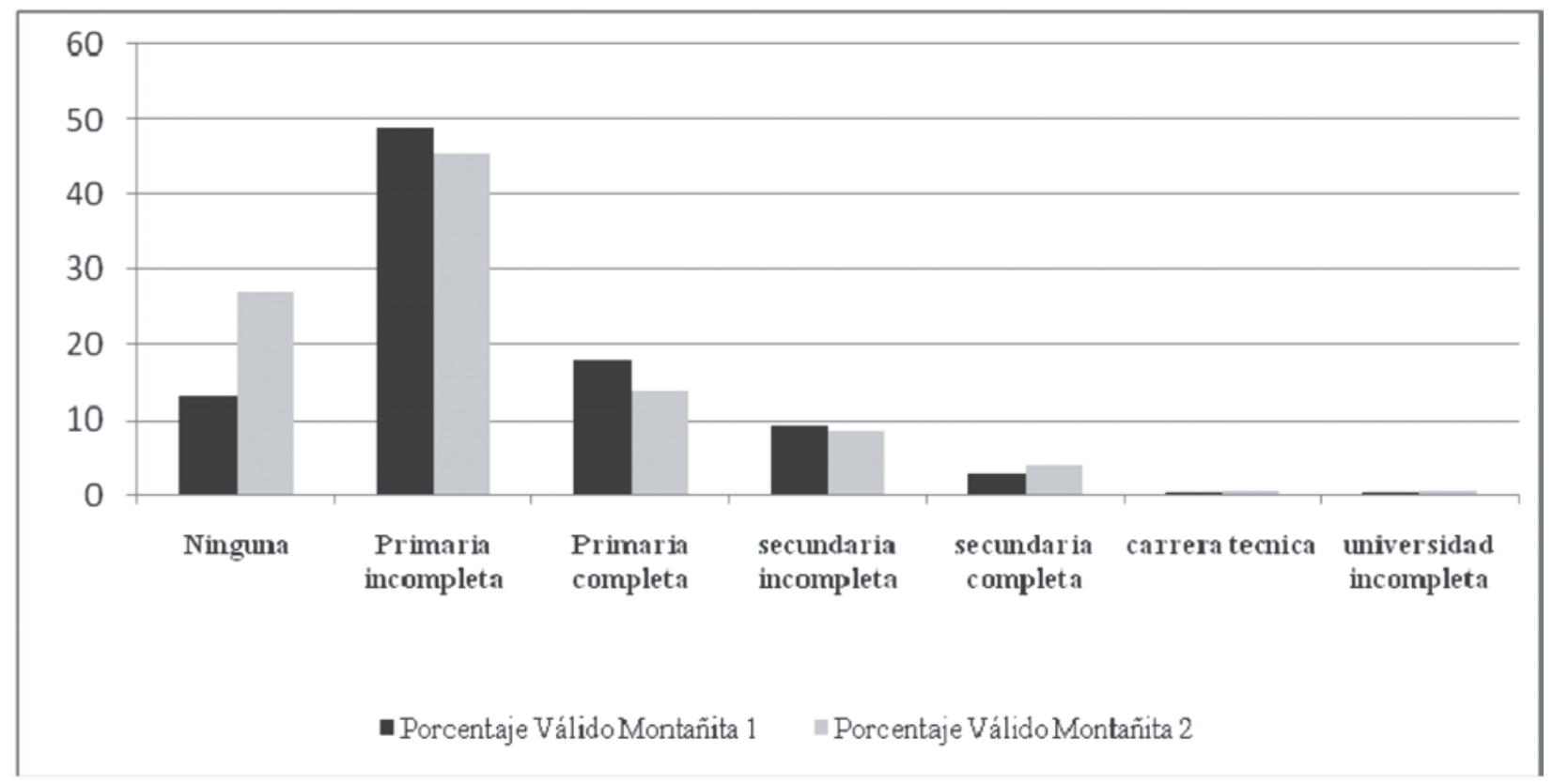

Figura 2. Distribución porcentual de la población de La Montañita 1 y 2 por nivel de escolaridad.

De manera que no se puede hablar de proyectos de erradicación de la pobreza, ni de empoderamiento de las comunidades sino incluyen estrategias precisas para mejorar los niveles de conocimiento de la población.

En el contexto de las comunidades estudiadas se encontraron poblaciones con altos índices de analfabetas, lo que representa un factor significativo a considerar en cualquier acción que se emprenda en pro del desarrollo y erradicación de la pobreza en el corto plazo.

El gráfico evidencia que en las comunidades rurales de La Montañita 1 y La Montañita 2, la mayor parte de la población tiene una formación de primaria incompleta potencialmente analfabetos; que un $13.2 \%$ de la población de La Montañita 1 y 27.1\% en La Montañita 2, nunca han recibido ningún tipo de formación.

Los datos del cuadro1 ayudan a comprender la situación en que se encuentra la educación en estas comunidades rurales de Tisma; el 60.7\% de la población (Montañita 1) dice que actualmente no estudia, teniendo una población de $66.5 \%$ hasta los 25 años, a esto hay que debido a que el $89.1 \%$ no ha recibido ningún tipo de capacitación y solamente el 10.9\% dice haber recibido.

Tabla 1. Estructura porcentual de la condición de escolaridad de la población encuestada en La Montañita 1 y 2

\begin{tabular}{llcc}
\hline \multicolumn{2}{l}{ Condición de escolaridad } & Montañita 1 & Montañita 2 \\
\hline Estudia & Sí & 39.3 & 28.8 \\
& No & 60.7 & 71.2 \\
\multirow{3}{*}{ Sabe leer } & Total & 100 & 100 \\
& Sí & 77.7 & 62.9 \\
& No & 22.3 & 37.1 \\
Sabe contar & Total & 100 & 100 \\
& Sí & 82.8 & 73.9 \\
\multirow{3}{*}{ Sabe escribir } & No & 17.2 & 26.1 \\
& Total & 100 & 100 \\
Total & No & 76.2 & 63.1 \\
& & 23.8 & 36.9 \\
& & 100 & 100
\end{tabular}




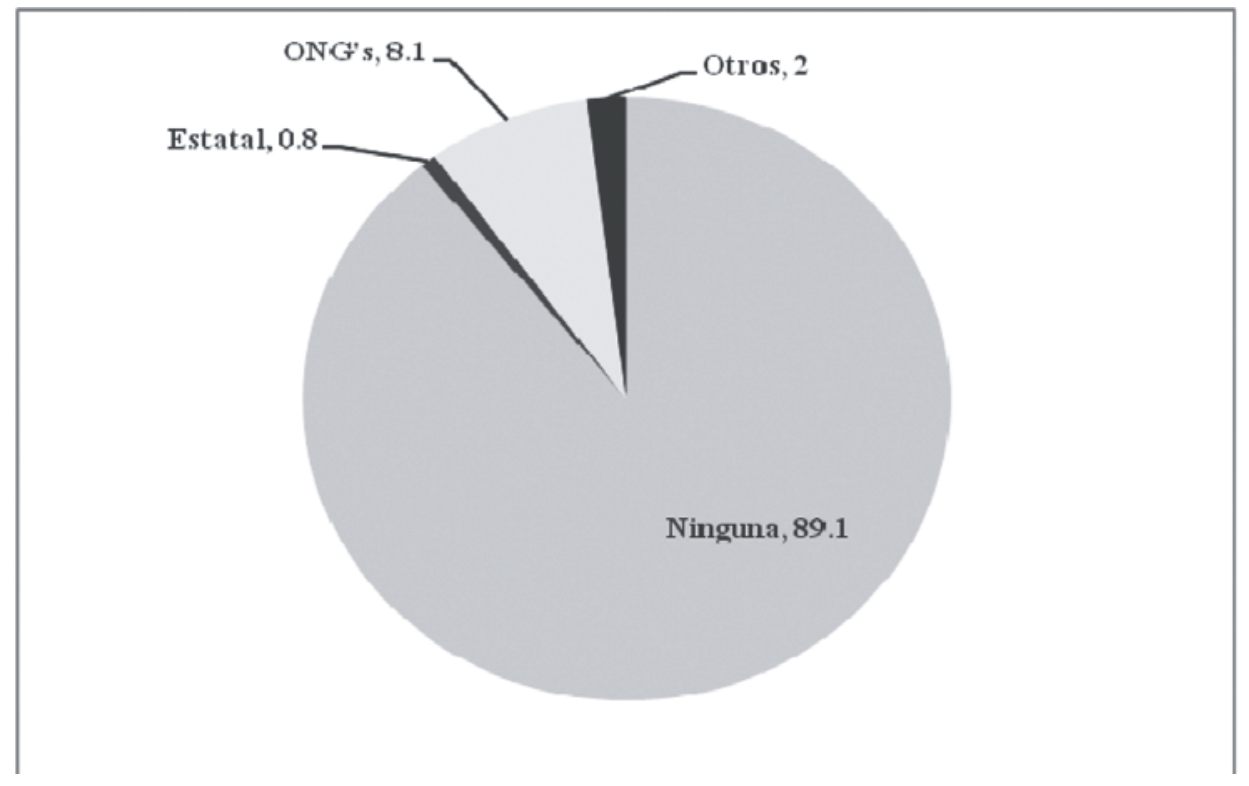

Figura 3. Porcentaje del tipo de instituciones que han brindado capacitaciones a la población encuestada de La Montañita 1 y 2.

Las personas que han recibido capacitación una sola vez representan el $4 \%$ de la población, esto indica que en estas comunidades los proyectos de desarrollo impulsados no han priorizado la capacitación como estrategia para el logro del mismo y por ende en el mejoramiento del nivel de vida.

Además, el 47.7\% de encuestados expresó que nadie
Un elemento importante que se determinó es que los tópicos de las pocas capacitaciones recibidas por los pobladores estaban relacionadas con la producción agrícola, lo cual representa una concepción de desarrollo vinculado a la producción agropecuaria a través de la implementación de nuevas o de adopción de tecnologías; es decir, que se percibe una concepción del desarrollo del

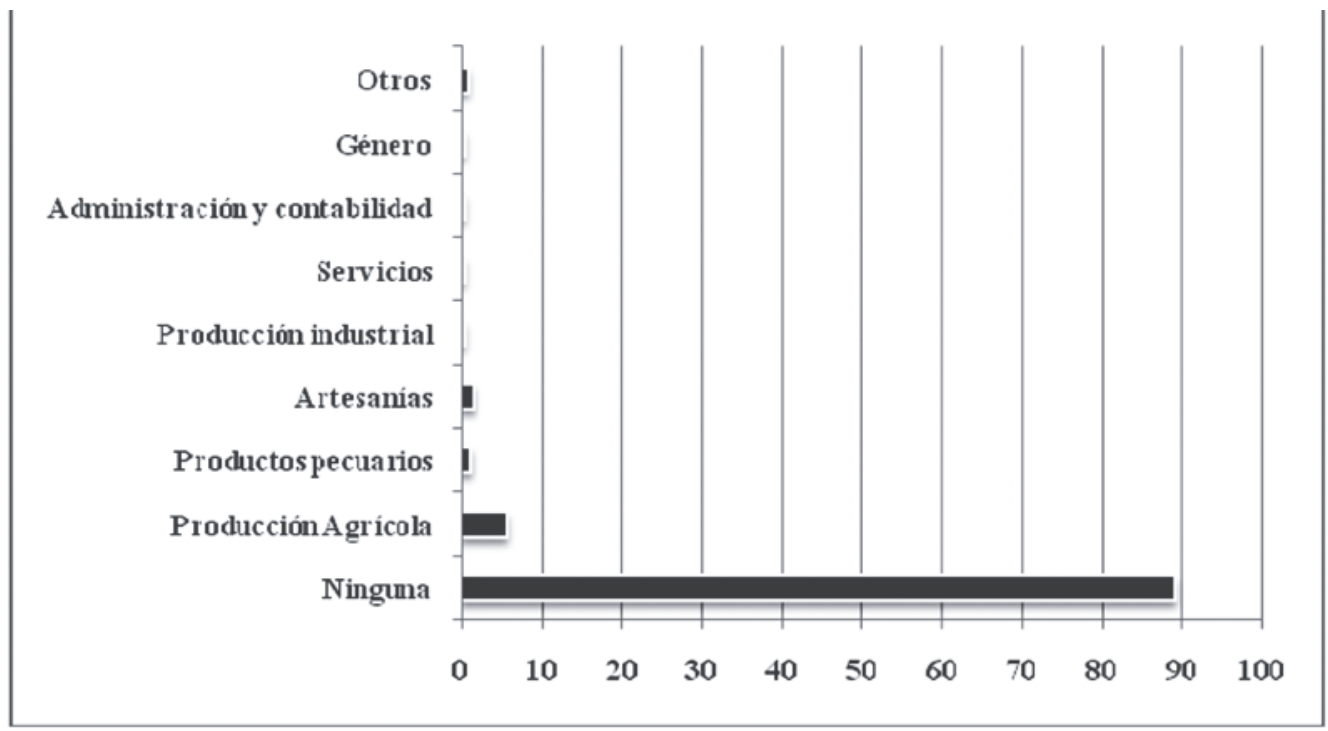

Figura 4. Peso porcentual de los diferentes temas de capacitaciones recibidas por la población de la montañita 1 y 2 encuestada.

ha llegado a capacitar a la comunidad y el $16.7 \%$ piensa que no tiene tiempo disponible para capacitarse, este sector agropecuario como el elevar el nivel tecnológico, lo que no se corresponde con las teorías relacionadas con la preponderancia del factor humano y el conocimiento como la esencia del desarrollo. 
Es importante mencionar que el 55.7\% de la población expresó no necesitar capacitación, lo que revela el grado de negatividad que se tiene acerca de la importancia de elevar sus niveles de conocimientos, constituyéndose en un indicativo de la necesidad de realizar esfuerzos para influenciar a estas comunidades en la aceptación y búsqueda de alternativas de programas de capacitación.

Nivel de organización comunitaria. Se menciona anteriormente que la organización comunitaria es un factor importante para el mejoramiento del nivel de vida de las comunidades rurales; Sin embargo se observó durante el estudio en las comunidades de La Montañita 1 y La Montañita 2, del municipio de Tisma que el 85.7\% no pertenece a ningún tipo de organización y solamente el 14.3\% tiene relación con alguna organización.

Se recomienda el considerar una estrategia de capacitación sobre la importancia de organizarse y el cómo organizarse, en función de sus necesidades más sentidas o aquellas situaciones que desfavorecen el desarrollo local; los pequeños productores de las comunidades rurales deben organizarse desde el punto de vista productivo, comercial, social y económico, para poder tener un mejor posicionamiento en el mercado y en el flujo de información.

Las organizaciones que mayor presencia tienen son los organismos no gubernamentales con un $12.3 \%$ de participación de la población de las comunidades estudiadas.

Una de las razones puntualizadas por la población para no organizarse es la politización de las organizaciones (17.1\%). Se recomienda trabajar en función de romper con estos esquemas y hacer que las comunidades recobren la confianza y comprendan las ventajas del estar organizado; por otro lado, se requiere un trabajo sostenido y coordinado de los distintos organismos e instituciones, dado que la confianza es un elemento que no se logra de forma inmediata ante una sólo acción asilada. Por otro lado, el 12.8\% población que expresa no tener tiempo es por la misma razón de no visualizar ninguna ventaja de estar organizado. Esta apatía representa una barrera para emprender acciones de desarrollo comunitario.

Todo esto queda demostrado cuando el $85.5 \%$ considera no haber recibido ningún beneficio de alguna organización, por lo que se infiere la necesidad de trabajar en dos direcciones: la visualización de la importancia de estar organizado y la de conceptualización de que los beneficios no pueden ser materiales o bien tangibles a lo inmediato; se deberá entonces priorizar el empoderamiento de las comunidades y eso tiene que ver con potenciar el conocimiento autóctono y el nivel formativo general.

Los datos de la tabla 2, evidencian que la principal actividad económica a la que se dedican es al cultivo

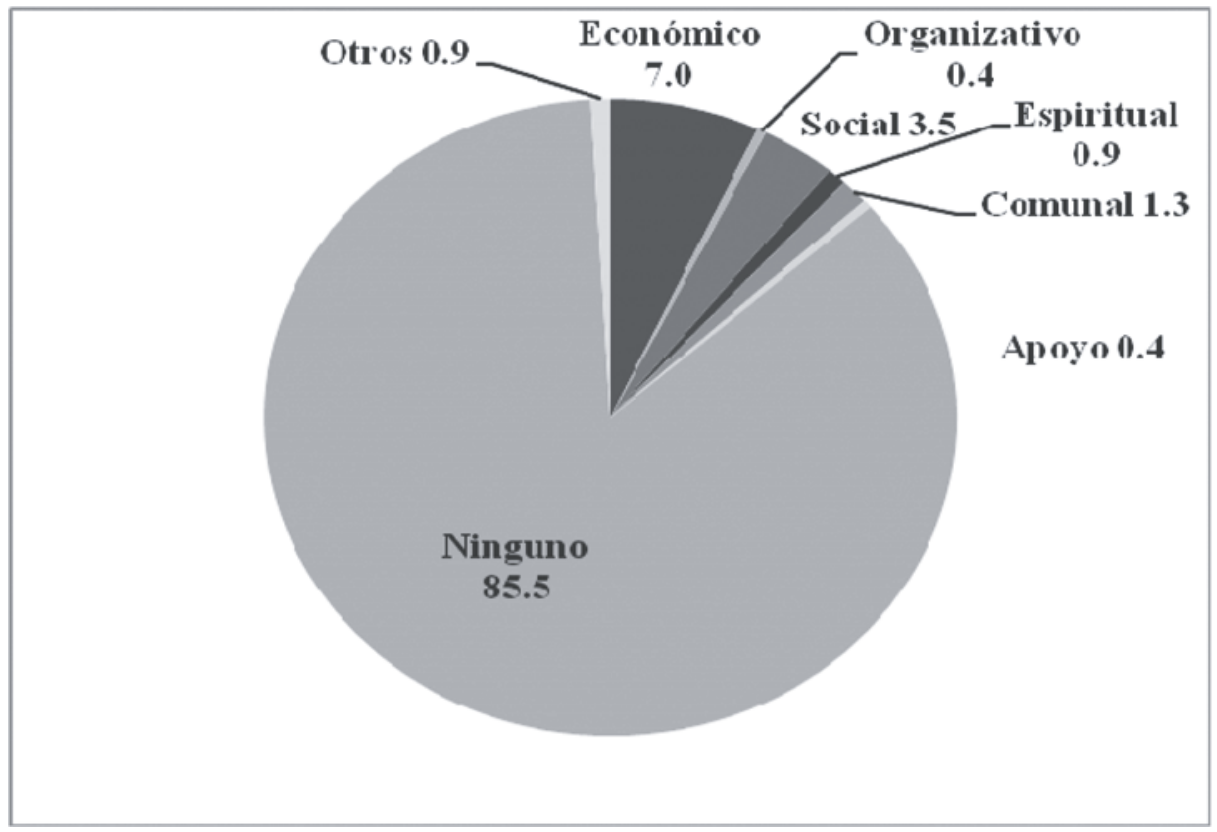

Figura 5. Peso porcentual de la opinión de los beneficios obtenidos por los participantes en proyectos de La Montañita 1 y 2. 
Tabla 2. Peso porcentual de las principales actividades económicas en las comunidades de La Montañita 1 y 2.

\begin{tabular}{lrr}
\hline Principal Actividad & Montañita 1 & Montañita 2 \\
\hline Cultivos & 30.2 & 36.6 \\
Ganadería & 1.3 & 1.6 \\
Cultivo/ganadería & 0.7 & $\mathrm{O}$ \\
Comercio & 5.4 & 4.1 \\
Servicios & 11.4 & 4.1 \\
Procesamiento & 1.3 & 0.8 \\
Artesanías & 8.7 & 14.6 \\
Industria & 0.7 & 0.8 \\
Doméstica & 4.7 & 0.8 \\
Hija de casa & 12.1 & 20.3 \\
Otra actividad & 23.5 & 16.3 \\
& & \\
\hline
\end{tabular}

(30.2\% y 36.6\%), en segundo lugar, apuntan que a cualquier tipo de trabajo que salga (23.5\% y $16.3 \%$.

Las otras actividades indican, de alguna manera, un grado de urbanización de las ocupaciones, tales como: un $11.4 \%$ se dedica a los servicios, 5.4\% al comercio, y el $8.7 \%$ a la artesanías, entre otras actividades de este mismo tipo.

La actividad que se requiere potenciar es la agricultura en toda su dimensión para obtener valor agregado: procesamiento, comercialización y tecnología.

\section{CONCLUSIONES}

Las familias se caracterizan por ser extensas, dado que conviven dos o más generaciones en el mismo grupo doméstico, con un promedio de siete personas por hogar.

La población es mayoritariamente joven, lo que implica que en la mayoría de los hogares una a dos personas trabaja.

Los ingresos promedio por familia están por debajo del costo de la canasta básica.

Los pobladores tienen un bajo nivel educativo.

La población tiene poco o casi nulo acceso a programas de capacitación.

Existe poca participación en las organizaciones comunitarias.

En la zona (Municipio) hay pocas oportunidades de empleo.

Existen falta de oportunidades y condiciones materiales, producto de la desigualdad social.

Todos estos aspectos determinan el nivel de pobreza de las comunidades de La Montañita 1 y 2 del Municipio de Tisma, Departamento de Masaya.

\section{REFERENCIAS BIBLIOGRÁFICAS}

AMUNIC (Asociación de Municipios de Nicaragua). 1997. Tisma Departamento de Masaya, Managua, Nicaragua.

FAO (Organización de las Naciones Unidas para la Agricultura y la Alimentación). 1999. La Juventud Contra el Hambre. Roma 23 p.

FNUAP (Fondo de Población de las Naciones Unidas). 1999. Estado de la población mundial: Resumen ejecutivo, Managua, Nicaragua.

FNUAP (Fondo de Población de las Naciones Unidas). 2001. Diagnóstico sociodemográfico, Municipio de Jinotega, Managua, Nicaragua.

INIFOM-GTZ. 1999. Plan indicativo de desarrollo municipal. Municipio de Tisma. Tomo N. 1. Managua, Nicaragua, $70 \mathrm{p}$.

INEC (Instituto Nacional de Estadísticas y Censo, NI). 2005. VIII Censo de Población y IV de Vivienda

INEC (Instituto Nacional de Estadísticas y Censo, NI). 1999. Encuesta de medición de vida, Managua, Nicaragua.

NITLAPAN - UCA. 1997. Apuntes para una estrategia de desarrollo rural, Managua, Nicaragua.

PNUD (Programa de las Naciones Unidas para el Desarrollo). 1998. Informe sobre Desarrollo Humano. Ediciones Mundi-Prensa, Madrid, España. Pág. 228

PNUD (Programa de las Naciones Unidas para el Desarrollo). 2000. Equidad para superar la vulnerabilidad, Managua, Nicaragua.

Toffler, A. 1990. El Cambio del Poder, P y J Editores. 